\title{
Método para la elaboración de la currícula, basado en competencias en la enseñanza de la física general en la Facultad de Ingeniería Química y Textil de la Universidad Nacional de Ingeniería
}

\author{
Method for the elaboration of the curriculum, based on competences \\ in the teaching of general physics in the Faculty of Chemical and \\ Textile Engineering of the National University of Engineering
}

\author{
Juan Sánchez ${ }^{1^{*}}$ \\ ${ }^{1}$ Facultad de Ingeniería Química y Textil de la Universidad Nacional de Ingeniería. Lima, Perú \\ Recibido (Recieved): 18/10/ 2018 \\ Aceptado (Accepted): 17/ 12/2018
}

\section{RESUMEN}

Se plantea una competencia de metodología educativa partiendo del tema "Leyes de la Termodinámica" del sílabo del curso Física II que se imparte a los estudiantes de la Facultad de Ingeniería Química y Textil de la Universidad Nacional de Ingeniería (UNI). Esta competencia aborda el desarrollo del proyecto formativo "Implementación de la cultura en termodinámica" que permite, a los estudiantes de la Facultad, interpretar y buscar soluciones a un problema de actualidad, como es la contaminación ambiental generada por el parque automotor de Lima. El desarrollo de la competencia propuesta implica: 1/ La elaboración de una matriz del proyecto, 2 / la constitución de un grupo de trabajo (4 estudiantes), 3/ la planificación de los trabajos colaborativos, 4/ el seguimiento de la reunión del grupo de trabajo, 5/ la entrega de informes, y 6/ la calificación de los trabajos colaborativos.

Palabras Clave: Leyes de la Termodinámica, competencias, contaminación ambiental, parque automotor de Lima.

\section{ABSTRACT}

It is proposed a competence of educational methodology starting from the subject "Thermodynamic Laws" of the syllabus of the Physics II course which is taught to the students of the Faculty of Chemical and Textile Engineering of the Universidad Nacional de Ingeniería (UNI). This competence approaches the development of the training project "Implementation of culture in thermodynamics", which allows the students of the Faculty to interpret and search solutions to a current problem, such as the environmental pollution generated by the car park in Lima. The development of the proposed competence involves the: 1 / elaboration of a matrix of the project, 2 / constitution of a working group (4 students), 3 / planning of the collaborative works, 4 / follow-up of the group meeting work, 5 / reporting, and 6 / qualification of the collaborative work.

Keywords: Thermodynamic laws, competence, environmental pollution, Lima car park.

\section{INTRODUCCION}

En la malla curricular de la formación profesional del Ingeniero Químico Industrial y Textil, la enseñanza de la Física General está basada en contenidos con metas concretas y se esperan que los estudiantes, al final de un proceso formativo, pongan en acción sus capacidades en torno a actividades o tareas puntuales que pueden ser del tipo cognitivo, afectivo y psicomotor. La malla curricular, basado en competencias, se apoya en contenidos para afrontar las necesidades de los contextos sociales con creatividad y comprensión. Para lograr los objetivos propuestos es importante desarrollar y aplicar, de manera articulada,
Ios siguientes saberes: saber ser, saber convivir, saber hacer y saber conocer. En este contexto se plantea el Proyecto Formativo titulado "IMPLEMENTACIÓN DE LA CULTURA EN TERMODINÁMICA" para interpretar los efectos de contaminación medioambiental generados por el Parque Automotor en Lima.

\section{OBJETIVO}

El objetivo principal de este estudio es hacer ver, cómo se aplica el criterio de las competencias para poder elaborar una currícula de enseñanza del curso de Física General II. Teniendo en cuenta las referencias [1 al 5], proponemos desarrollar la competencia de la siguiente

\footnotetext{
* Corresponding author.:
}

E-mail: jcdabi@hotmail.com 
manera: 1/ Elaboración de la competencia; 2/ Localizar el problema del contexto; 3/Transversalidad; 4/ Criterios y evidencias; $5 /$ Tareas.

\section{MATERIALES}

- Sílabo del curso de Física General II.

- Materiales bibliográficos sobre temas de calor y trabajo, primera y segunda ley de la termodinámica, procesos y ciclos termodinámicos, ciclo de Carnot, ciclo de Otto y de Diesel.

- Manuales y Catálogos sobre motores de combustión interna.

- Estudios sobre la contaminación del aire atmosférico en Lima realizados por: Servicio Nacional de Meteorología e Hidrología del Perú (SENAMHI), Ministerio de Transportes y Comunicaciones (MTC) y el Ministerio de Salud (MINSA).

\section{EVIDENCIAS}

En la Figura 1 se muestra la UVE Heurística del problema a estudiar aplicando la Termodinámica y la Teoría de las máquinas térmicas de combustión interna.

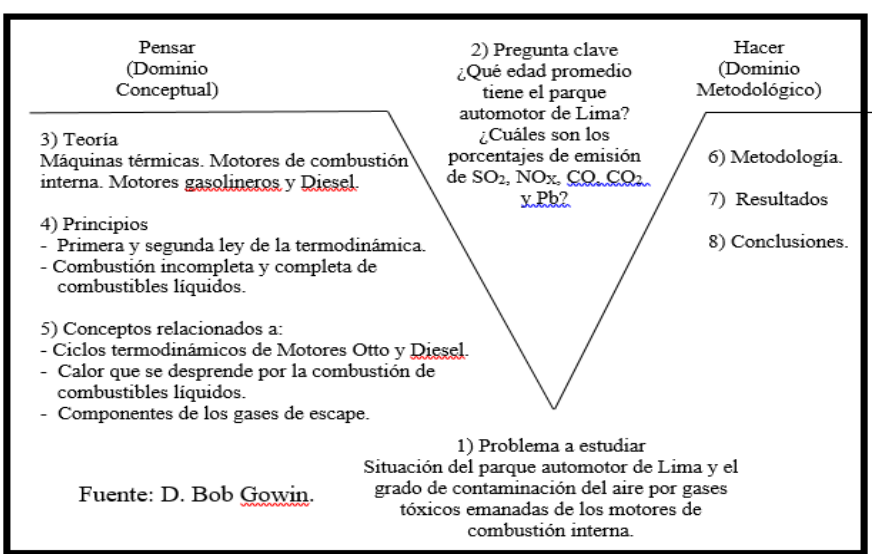

Figura 1. Heurística del problema

\section{DOMINIO CONCEPTUAL}

\section{TEORÍA}

Los equipos energéticos que más aceptación han tenido en los medios de transporte han sido los motores alternativos de combustión interna. La peculiaridad de estos motores consiste en que el proceso de combustión de la mezcla combustible con el aire y la transformación de su correspondiente energía en mecánica tiene lugar dentro del cilindro del motor. El uso del petróleo como combustible líquido, a finales del siglo XIX, estimuló la creación y producción de motores de combustión interna: motores con carburador de encendido por chispa (gasolineros), motores de encendido por incandescencia o semi-diesel y motores de encendido por compresión (Diesel).

\section{PRINCIPIOS}

- Primera ley de la Termodinámica: La energía no se crea ni se destruye, sólo se transforma. Es decir la energía puede adoptar diferentes formas, como puede ser calor, trabajo, energía cinética, potencial, etc. La expresión matemática es [6]:

$$
\oint \delta Q=\oint \delta W
$$

- Segunda Ley de la Termodinámica: existen muchas formas de enunciar esta ley. Citaremos los enunciados de Kelvin - Planck y Clausius.

Enunciado de Kelvin - Planck: "Es imposible construir una máquina térmica que, operando continuamente (cíclico) transforme íntegramente el calor que recibe de una fuente térmica, a temperatura uniforme, en trabajo".

Enunciado de Clausius: "El calor no se transmite nunca espontáneamente hacia otra cuya temperatura sea mayor" [6].

- La combustión completa de combustibles líquidos [7] incluyen reacciones como las descritas en las reacciones (2) y (3)

$$
\begin{gathered}
\mathrm{C}+\mathrm{O}_{2}=\mathrm{CO}_{2} \\
2 \mathrm{H}_{2}+\mathrm{O}_{2}=2 \mathrm{H}_{2} \mathrm{O} \text { (vapor) }
\end{gathered}
$$

La cantidad mínima de oxígeno necesario para que $1 \mathrm{Kg}$ de combustible se queme totalmente es:

$$
O_{\text {min }}=\frac{8}{3} \mathrm{C}+8 \mathrm{H}-\mathrm{O}_{\mathrm{C}}
$$

donde $O_{c}$ es el contenido de Oxígeno en el combustible. La cantidad de aire (que contiene $23 \%$ en masa de oxígeno) necesario para la combustión de $1 \mathrm{Kg}$ de combustible viene dada por la siguiente relación:

$$
L_{0}=\frac{1}{0,23}\left[\frac{8}{3} \mathrm{C}+8 \mathrm{H}-\mathrm{O}_{\mathrm{C}}\right] \quad(\mathrm{Kg})
$$

- La combustión incompleta de los combustibles líquidos, incluye la siguiente reacción, [7]

$$
2 \mathrm{C}+\mathrm{O}_{2}=2 \mathrm{CO}
$$

Si $\varphi$ es la fracción de combustible que al quemarse forma CO, la masa de este compuesto quemado es $\varphi C$ $(\mathrm{Kg})$, mientras la masa de $\mathrm{CO}_{2}$ es $(1-\varphi) \mathrm{C}(\mathrm{Kg})$. La cantidad de aire necesario para la combustión incompleta de $1 \mathrm{Kg}$ de combustible es:

$$
L_{o}=\frac{1}{0,23}\left[\frac{4}{3}(2-\varphi) C+8 H-O_{C}\right]
$$

\section{CONCEPTOS}

\section{Ciclos termodinámicos}

CICLO OTTO (Gasolinero): Es el ciclo idealizado de los motores a gasolina o de encendido por chispa y está compuesto de cuatro procesos. Comprensión isoentrópica, combustión a volumen constante, 
expansión isoentrópica y expulsión de gases a volumen constante. Además de estos procesos es necesario considerar dos carreras adicionales de pistón del motor que corresponden a la admisión de la mezcal aire combustible y la de barrido de los gases.

Eficiencia del Ciclo OTTO:

$$
\eta_{\mathrm{t}}=1-\frac{1}{\epsilon^{K-1}}
$$

$\in$ : Relación de compresión $(6-9.5) ; \mathrm{K}($ aire $)=$ 1,3

CICLO DIESEL (Petrolero): La diferencia fundamental de este ciclo con el ciclo Otto radica en el hecho que durante la admisión el motor absorbe únicamente aire, que es comprimido hasta alcanzar una temperatura por encima de la correspondiente a la autoignición del combustible, de tal forma que si al final de la compresión se inyecta combustible a la mezcla éste se encenderá automáticamente. La combustión se realiza a presión constante.

Eficiencia del Ciclo DIESEL:

$$
\eta_{\mathrm{t}}=1-\frac{1}{\epsilon^{K-1}} \cdot \frac{\rho^{K}-1}{K(\rho-1)}
$$

$\rho$ : Grado de expansión previa (Relación de combustión a presión constante)

Eficiencia térmica de una máquina térmica $(\eta)$ :

$$
\begin{gathered}
\eta=\frac{\text { Trabajo realizado por la máquina }}{\text { Calor recibido por la máquina }} \\
0 \quad \begin{array}{c}
<<1
\end{array}
\end{gathered}
$$

Calor que se desprende por la combustión de combustibles líquidos [7]

Combustión completa. Se puede calcular utilizando la fórmula:

$$
\mathrm{H}_{\mathrm{U}}=\mathrm{H}_{\mathrm{O}}-(2512+9 \mathrm{H}+\mathrm{W}) \quad\left(\frac{K J}{K g}\right)
$$

Siendo Ho, el calor total desprendido durante la combustión (kJ). La cantidad de calor necesario para generar $1 \mathrm{Kg}$ de vapor de agua es $2512 \mathrm{~kJ}$. 9H es la cantidad de vapor de agua que se forma al arder $1 \mathrm{Kg}$ de combustible, siendo $\mathrm{H}$, la fracción de masa de hidrógeno que contiene $1 \mathrm{~kg}$ de combustible. $W$ es la cantidad de humedad que hay en $1 \mathrm{Kg}$ de combustible.

Componentes de los gases de escape. Definamos el coeficiente de exceso de aire $(\alpha)$

$$
\alpha=\frac{l}{l o}
$$

Siendo l la cantidad teórica de aire necesario para la combustión de $1 \mathrm{Kg}$ de combustible; lo la cantidad real de aire necesario para la combustión de $1 \mathrm{Kg}$ de combustible. Motores Otto: $\alpha: 0,85-1,15$ y Motores Diesel: $\alpha: 1,3-5$ Los componentes tóxicos de los gases de escape son:

i/ Monóxido de carbono (CO) cuando $a<1$ (combustión incompleta la cantidad de CO puede alcanzar: 10 - 12\% (en volumen) del total de los productos de la combustión.

ii/ El óxido nítrico (NO) y el dióxido de nitrógeno $\left(\mathrm{NO}_{2}\right)$ se encuentran en pequeña cantidad $(0,8 \mathrm{mg} / \mathrm{l})$.

iii/ El anhídrido sulfuroso $\left(\mathrm{SO}_{2}\right)$ y el ácido sulfhídrico $\mathrm{H}_{2} \mathrm{~S}$, se forman cuando el motor funciona con combustibles que contienen azufre. La cantidad de $\mathrm{SO}_{2}$ puede llegar hasta $250 \mathrm{mg} / \mathrm{l}$. La $\mathrm{H}_{2} \mathrm{~S}$ es insignificante.

Recomendaciones técnicas para mejorar la eficiencia térmica en las máquinas térmicas [8]: Motores Otto (automóviles), RPM en el eje: 3500;

Carga, 75\% de la plena carga (máxima potencia). Motores Diesel (Camiones Petroleros), RPM en el eje: 1500 ; Carga, $75 \%$ de la plena carga.

\section{DOMINIO METODOLÓGICO}

\section{METODOLOGÍA}

\section{.}


Elaboración de una matriz, al que llamamos Proyecto formativo I y que se describe a continuación,

\begin{tabular}{|c|c|c|}
\hline \multicolumn{2}{|c|}{$\begin{array}{ll}\text { PROYECTO FORMATIVO I } \\
\end{array}$} & \\
\hline \multirow{5}{*}{\multicolumn{2}{|c|}{$\begin{array}{l}\text { ÁREA DE FORMACIÓN: FÍSICA GENERAL II } \\
\text { PROYECTO FORMATIVO I: IMPLEMENTACIÓN DE LA CULTURA EN } \\
\text { TERMODINÁMICA PARA INTERPRETAR LA SITUACIÓN DEL PARQUE AUTOMOTOR } \\
\text { DE LIMA. } \\
\text { CONTENIDOS ABORDADOS: } \\
\text { - Calor y trabajo } \\
\text { - Primera y segunda ley de la termodinámica } \\
\text { - Procesos y ciclos termodinámicos } \\
\text { - Ciclo de Carnot } \\
\text { - Ciclo Otto y Diessel. }\end{array}$}} & $\begin{array}{l}\text { CURSO: Física II - FI204 } \\
\text { DURACIÓN: } 04 \text { semanas. }\end{array}$ \\
\hline & & DOCENTE: Ing. Juan Ignacio Sánchez Dávalos \\
\hline & & $\begin{array}{l}\text { ESTRATEGIA DIDÁCTICA: } \\
\text { - Lección magistral } \\
\text { - Gestión de Provectos }\end{array}$ \\
\hline & & $\begin{array}{l}\text { - Trabajo de campo y laboratorio } \\
\text { - Trabajo colaborativo. }\end{array}$ \\
\hline & & \begin{tabular}{ll|} 
& FECHA DE ENTREGA \\
- Revisión del diseño: \\
- \\
- & Entrega del informe: \\
\end{tabular} \\
\hline \multicolumn{3}{|c|}{$\begin{array}{l}\text { COMPETENCIA QUE SE VA A CONTRIBUIR A FORMAR: Gestiona proyectos creativos e innovadores que permitan a tener una cultura termodinámica, } \\
\text { para poder interpretar la situación del Parque Automotor de Lima, para luego proponer soluciones alternativas que se adecuen a la realidad aplicando la } \\
\text { termodinámica y la teoria de máquinas de combustión interna. }\end{array}$} \\
\hline \multirow{2}{*}{\multicolumn{3}{|c|}{$\begin{array}{l}\text { PROBLEMA DE CONTEXTO: ¿Cómo implementar una cultura en la termodinámica, para poder interpretar la situación del Parque Automotor de Lima y } \\
\text { proponer soluciones a los problemas como la contaminación del aire atmosférico, el tránsito vehicular entre otros?. } \\
\text { TRANSVERSALIDAD: Parque Automotor de Lima, Ministerio de Transportes y Comunicaciones (MTC), Servicio Nacional de Meteorología e Hidrología del } \\
\text { Perú (SENAMHI) }\end{array}$}} \\
\hline & & \\
\hline $\begin{array}{l}\text { CRITERIOS: } \\
\text { 8. Trabaja de manera colaboradora para la gestión de información de la } \\
\text { situación del Parque Automotor de Lima. } \\
\text { 9. Diagnostica la contaminación del aire atmosférico debido a los gases de } \\
\text { escape, producto de la combustión. } \\
\text { 10. Elabora Informes Técnicos de la contaminación del aire atmosférico, } \\
\text { según las normas de la Organización Mundial de la Salud (OMS). } \\
\text { 11. Gestiona recursos, seminarios, convenciones con el propósito de } \\
\text { implementar un proyecto de cultura en la termodinámica para el } \\
\text { diagnóstico del Parque Automotor de Lima. }\end{array}$ & \multicolumn{2}{|c|}{$\begin{array}{l}\text { EVIDENCIAS: } \\
\text { 1. UVE Heuréstica del problema a estudiar aplicando los conocimientos de la } \\
\text { termodinámica y las máquinas de combustión interna. } \\
\text { 2. Informe de ejecución del proyecto. } \\
\text { 3. Registro del trabajo colaborativo en el proyecto. }\end{array}$} \\
\hline ( & ACIÓN D & PARQUE AUTOI \\
\hline
\end{tabular}

Instalación de un grupo de trabajo de 4 estudiantes: Ver formato 01

\section{FORMATO 01}

FORMATO DE INSTALACIÓN DE GRUPO DE TRABAJO (Este formato debe ser llenado solo una vez por ciclo y en el se deben detallar las consideraciones de cómo se van a trabajar: hora, fecha y lugar de la reunión, disponibilidad de la logistica computadora internet, laptops, correos teléfonos, reglas de juego lo más detallado posible, planes de contingencia, análisis FODA de los integrantes, etc.)

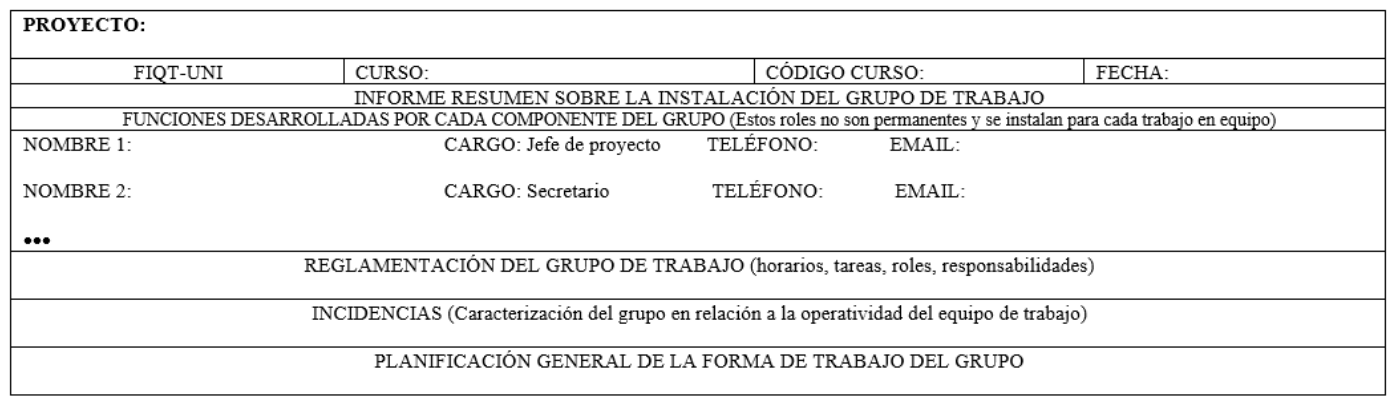

$\mathrm{V}^{\circ} \mathrm{B}^{\circ}$

Fecha:

Planificación de los trabajos colaborativos por cada proyecto. Ver formato 02.

FORMATO 02

PLANIFICACIÓN DE LOS TRABAJOS COLABORATIVOS (Este formato se llena una vez por cada trabajo. Donde se planifíca antes de inicia el trabajo todo lo que se tiene que resolver: la logística la distribución de tareas, el liderazgo y las responsabilidades las estrategias para resolver cada una de los problemas, fechas y plazos junto con los responsables para abordar cada problema a resolver, hasta la entrega final considerando todas las contingencias posibles.

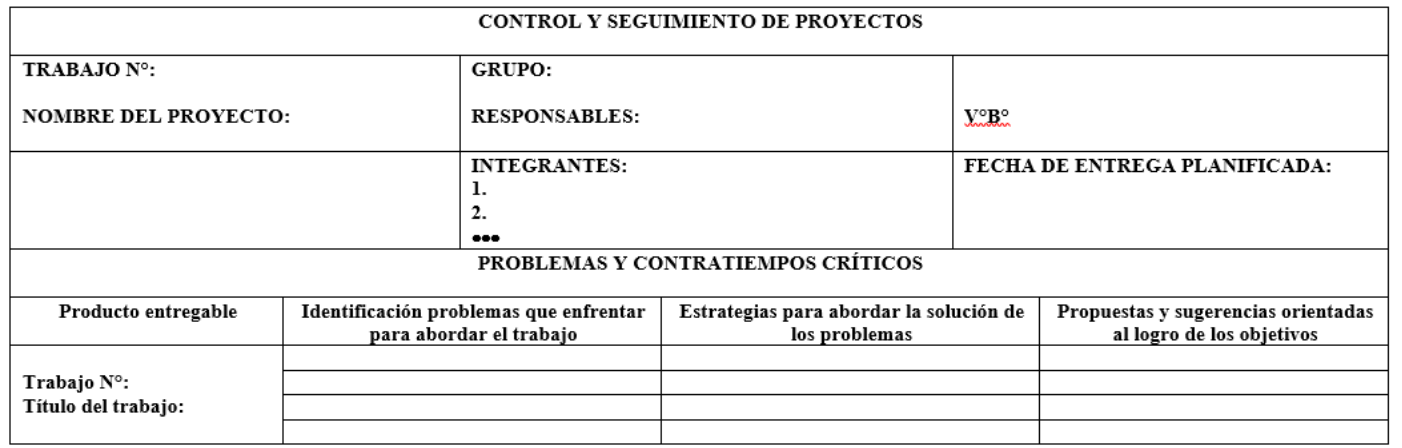


Control y seguimiento del proyecto: Ver formato 03.

\section{FORMATO 03}

FORMATO DE INCIDENCIAS DE LAS REUNIONES DE LOS GRUPOS DE TRABAJO (cada sesión de trabajo se entrega este formato donde se debe considerar todas las incidencias de la reunión, faltas, tardanzas, incumplimientos, contingencias Análisis FODA de los integrantes, logros en la solución de problemas, cumplimiento en la socialización del aprendizaje, (¿todos aprendieron?) y la planificación de las tareas para la siguiente reunión)

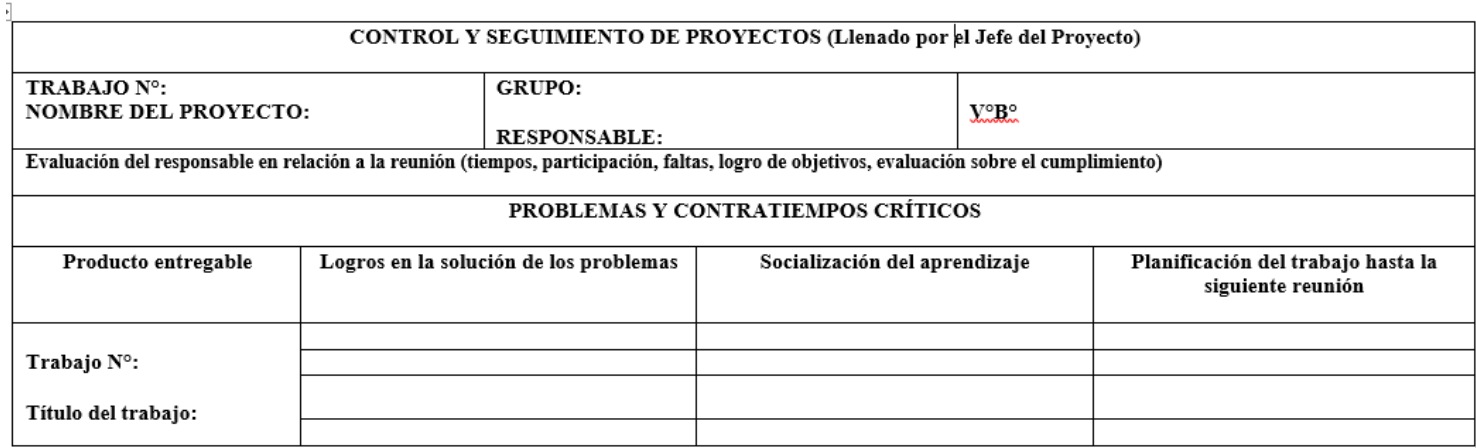

Entrega del informe: Ver formato 04.

\section{FORMATO 04}

FORMATO DE ENTREGA DEL INFORULE. (solo se llena un formato por cada trabajo entregado. Si el trabajo implica varias tareas en este formato se resume todas las incidencias para todas las tareas del trabajo)

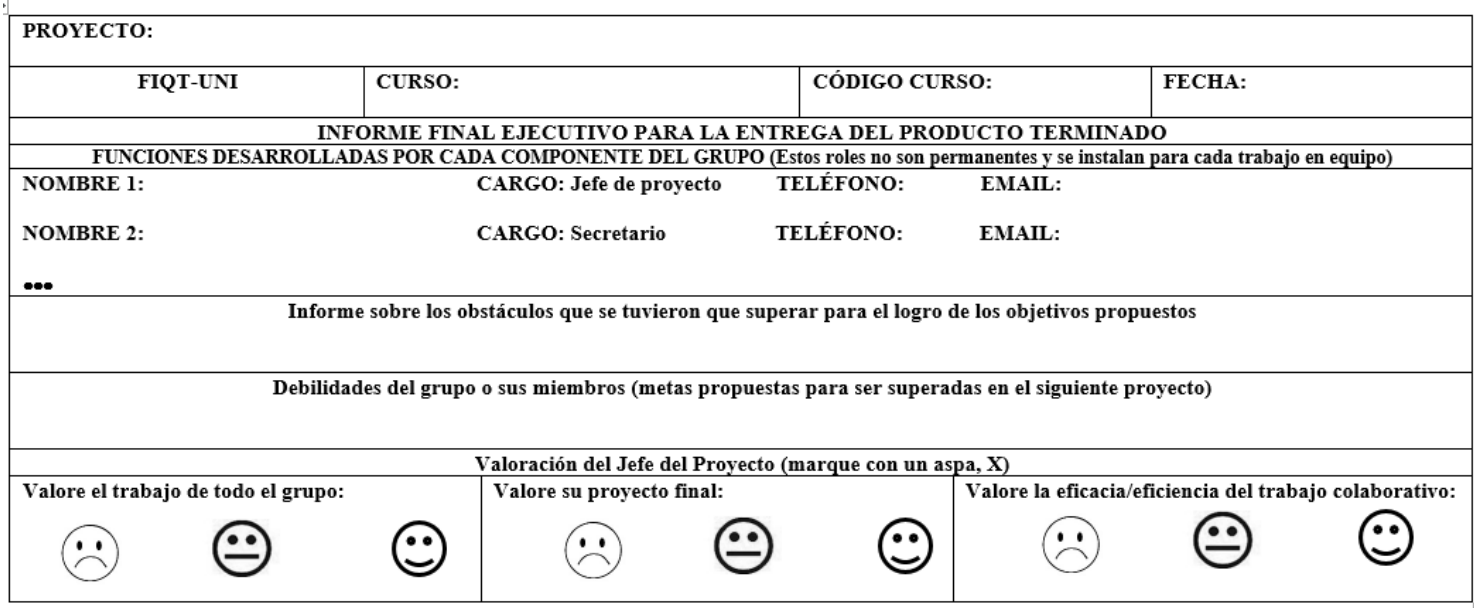

$\mathrm{Y}^{\circ} \mathbf{B}^{\circ}$ :

Fecha: 
Valoración global del informe. Ver anexo 05.

\begin{tabular}{|c|c|c|c|c|c|}
\hline \multicolumn{6}{|c|}{$\begin{array}{l}\text { ANEXO 05 } \\
\end{array}$} \\
\hline CRITERIO & $\begin{array}{c}\text { Sin competencia } \\
\mathbf{3 5} \%\end{array}$ & $\begin{array}{c}\text { Deficiente } \\
\mathbf{5 0 \%}\end{array}$ & Insuficiente $60 \%$ & \begin{tabular}{|c|}
$\begin{array}{c}\text { En proceso de adquirir la } \\
\text { competencia } 80 \%\end{array}$ \\
\end{tabular} & Competente $100 \%$ \\
\hline $\begin{array}{c}\text { Tiempos de } \\
\text { entrega }\end{array}$ & No entrego el informe & $\begin{array}{l}\text { Informe incompleto con } \\
\text { entrega a destiempo }\end{array}$ & $\begin{array}{l}\text { Informe completo con } \\
\text { entrega a destiempo } \\
\text { (más de } 1 \text { dia de retraso) }\end{array}$ & $\begin{array}{l}\text { Informe completo con un } \\
\text { retraso d e1 dia }\end{array}$ & $\begin{array}{l}\text { Informe completo con } \\
\text { entrega a tiempo }\end{array}$ \\
\hline Estructura de informe & No entrego el informe & $\begin{array}{c}\text { Estructura propia e } \\
\text { inadecuada de los autores } \\
\text { sin seguir los } \\
\text { lineamientos establecidos } \\
\end{array}$ & $\begin{array}{c}\text { Estructura propia y } \\
\text { adecuada de los autores } \\
\text { sin seguir los } \\
\text { lineamientos establecidos }\end{array}$ & $\begin{array}{l}\text { Estructura adecuada siguiendo } \\
\text { más del } 80 \% \text { de los } \\
\text { lineamientos establecidos }\end{array}$ & $\begin{array}{l}\text { Estructura adecuada } \\
\text { siguiendo el } 100 \% \text { de los } \\
\text { lineamientos establecidos }\end{array}$ \\
\hline $\begin{array}{l}\text { Normas APA de } \\
\text { redacción }\end{array}$ & No entrego el informe & $\begin{array}{l}\text { Informe con estilo propio } \\
\text { sin reflejar las normas } \\
\text { APA }\end{array}$ & $\begin{array}{l}\text { Informe siguiendo otras } \\
\text { normas de redacción }\end{array}$ & $\begin{array}{l}\text { Informe que sigue más del } 80 \% \\
\text { de la estructura propuesta por } \\
\text { normas APA }\end{array}$ & $\begin{array}{l}\text { Informe que sigue el } \\
100 \% \text { de la estructura } \\
\text { propuesta por normas } \\
\text { APA }\end{array}$ \\
\hline Trabajo colaborativo & No entrego el informe & $\begin{array}{l}\text { El trabajo ha sido } \\
\text { desarrollado por algunos } \\
\text { miembros del grupo }\end{array}$ & $\begin{array}{l}\text { El trabajo ha sido } \\
\text { desarrollado en forma } \\
\text { grupal }\end{array}$ & $\begin{array}{l}\text { El trabajo se ha desarrollado en } \\
\text { forma colaborativa con la } \\
\text { participación de la mayoria de } \\
\text { los miembros del grupo }\end{array}$ & \begin{tabular}{|} 
El trabajo se ha \\
desarrollado en forma \\
colaborativa con la \\
participación de todos los \\
miembros de grupo
\end{tabular} \\
\hline \multicolumn{2}{|c|}{$\begin{array}{l}\text { Valoración global del informe } \\
\text { (Promedio) }\end{array}$} & \multicolumn{4}{|c|}{$\begin{array}{l}\text { nota final } \\
=\underline{\text { tiempo entrega }+ \text { estructura informe }+ \text { normas } A P A+3 \text { trabajo colaborativo }}\end{array}$} \\
\hline
\end{tabular}

\section{RESULTADOS}

\section{Parque automotor de Lima}

De las emisiones atmosféricas relevantes que afectan a millones de personas que viven en la capital, las del parque automotor que circula diariamente por sus vías - el mayor del país - es la fuente más importante. Las estaciones de medición pública y privada monitorean las cantidades de contaminantes presentes en diversos puntos de la ciudad y nos permiten formular indicadores de calidad del aire.

El parque automotor del país, en términos globales es antiguo, pues aproximadamente el 80\% de los vehículos ingresados al país vía CETICOS y ZOFRATACNA, son usados y reacondicionados, influenciando en la edad promedio del parque automotor del servicio público que es 22.5 años, mientras del privado es 15.5 años, lo que ha generado el agravamiento de la contaminación ambiental, superando los estándares internacionales que miden la calidad del aire.

A fin de contribuir a revertir esta situación, se ha creado el Programa para la Renovación del Parque Automotor (chatarreo), inicialmente con vehículos de la categoría M1 con más de 15 años de antigüedad. Asimismo se ha establecido (D.S. N ${ }^{\circ}$ 017-2009-MTC) que la antigüedad máxima de los vehículos de transporte público es de 15 años. Cabe resaltar que en los últimos cinco años la importación de vehículos nuevos viene superando la importación de los vehículos usados (MTC). Según la Oficina General de Planificación y Presupuestos del Ministerio de Transportes y Comunicaciones, el parque automotor de Lima se estima en 1752919 vehículos el año 2016 como se indica en la Tabla 1. En la Tabla 2 se indica la evolución del parque automotor de Lima desde el año 2007-2016. La Figura 1 muestra la evolución del parque vehicular estimado a nivel nacional.

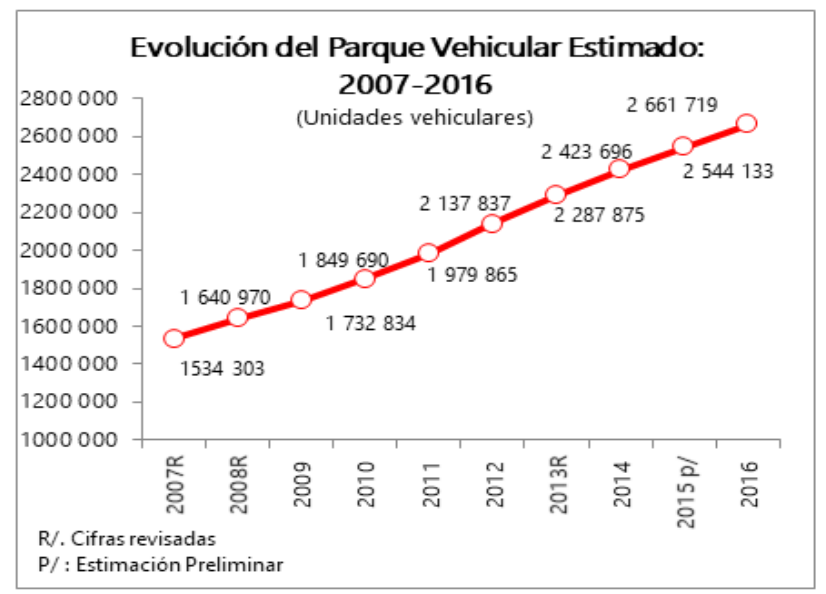

Figura 2. Evolución del parque automotor peruano 
Tabla 1. Parque Automotor Nacional en 2016 estimado por clase de vehículo, en Lima (y Callao) y el total nacional. Fuente: MTC - OGPP - OFICINA DE ESTADISTICA

\begin{tabular}{|c|c|c|c|c|c|c|c|c|c|c|}
\hline \multicolumn{11}{|c|}{ CLASE DE VEHICULO } \\
\hline \multirow{2}{*}{ Departamento } & \multirow{2}{*}{ TOTAL } & \multirow{2}{*}{ Automovil } & \multirow{2}{*}{$\begin{array}{l}\text { Station } \\
\text { Wagon }\end{array}$} & \multicolumn{3}{|c|}{ Camionetas } & \multirow{2}{*}{ Omnibus } & \multirow{2}{*}{ Camión } & \multirow{2}{*}{$\begin{array}{l}\text { Remol- } \\
\text { cador }\end{array}$} & \multirow{2}{*}{$\begin{array}{l}\text { Remolque } \\
\text { Semi-Rem. }\end{array}$} \\
\hline & & & & Pick Up & Rural & Panel & & & & \\
\hline $\operatorname{Lima*}$ & 1752919 & 807529 & 284251 & 163793 & 236502 & 31006 & 50441 & 116601 & 29520 & 33276 \\
\hline Total Nacional & 1752919 & 807529 & 284251 & 163793 & 236502 & 31,006 & 50,441 & 116,601 & 29,520 & 33276 \\
\hline
\end{tabular}

Tabla 2. Parque Vehícular Estimado, en Lima (y Callao) y el total nacional (2007-2016). Fuente: Superintendencia Nacional de los Registros Públicos (SUNARP)

$\mathrm{R} /$. Cifras revisadas, reajustadas por haberse detectado mayor incremento de inscripciones vehiculares.

$\mathrm{P} /$ : Estimacion Preliminar.

(Unidades Vehiculares)

\begin{tabular}{ccccccccccc}
\hline DPART AMENTO & $2007^{R}$ & $2008^{R}$ & 2009 & 2010 & 2011 & 2012 & $2013^{R}$ & 2014 & $2015^{p}$ & 2016 \\
LimayCallao & 957368 & 1036850 & 1106444 & 1195353 & 1287454 & 1395576 & 1498037 & 1590755 & 1674145 & 1752919 \\
TOTAL NACIONAL & 1534303 & 1640970 & 1732834 & 1849690 & 1979865 & 2137837 & 2287875 & 2423696 & 2544133 & 2661719 \\
\hline
\end{tabular}

\section{Contaminantes permitidos en el aire para Lima Metropolitana}

En el Perú, diferentes organismos y entidades se encargan de realizar estudios para verificar los niveles de contaminación existentes y su evolución con el tiempo. Dentro de estas entidades encontramos al Servicio Nacional de Meteorología e Hidrología del Perú (SENAMHI), el Ministerio del Medio Ambiente (MINAM), el Ministerio de Salud (MINSA), el Instituto Nacional de Estadística e Informática (INEI), Ministerio de Transportes (MTC) y diferentes organizaciones que aúnan sus investigaciones para llegar a conclusiones y datos muchos más específicos. El SENAMHI es uno de los 188 servicios meteorológicos reconocidas por la Organización Metereológica Mundial (OMM), por lo cual efectúa las acciones correspondientes en mérito a los convenios sobre composición atmosférica como el convenio de Viena para la protección de la capa de ozono (1985) y la Convención marco de las Naciones Unidas sobre el cambio climático (1994).

Entre los principales resultados, se destaca que el mayor problema de contaminación nacional se da en Lima Metropolitana donde son muy elevadas las concentraciones de material particulado menor a 10 micrómetros (PM10), dióxido de azufre $\mathrm{SO}_{2}$, dióxido de Nitrógeno $\left(\mathrm{NO}_{2}\right)$ monóxido de nitrógeno (NO), óxidos de nitrógeno $\left(\mathrm{NO}_{\mathrm{X}}\right)$ y ozono superficial $\left(\mathrm{O}_{3}\right)$. Cabe destacar que en el distrito de Ate y en 2011 la concentración de PM10 sobrepasaba en 48 veces el Estándar de Calidad Ambiental (ECA) (Ver Fig. 2). 


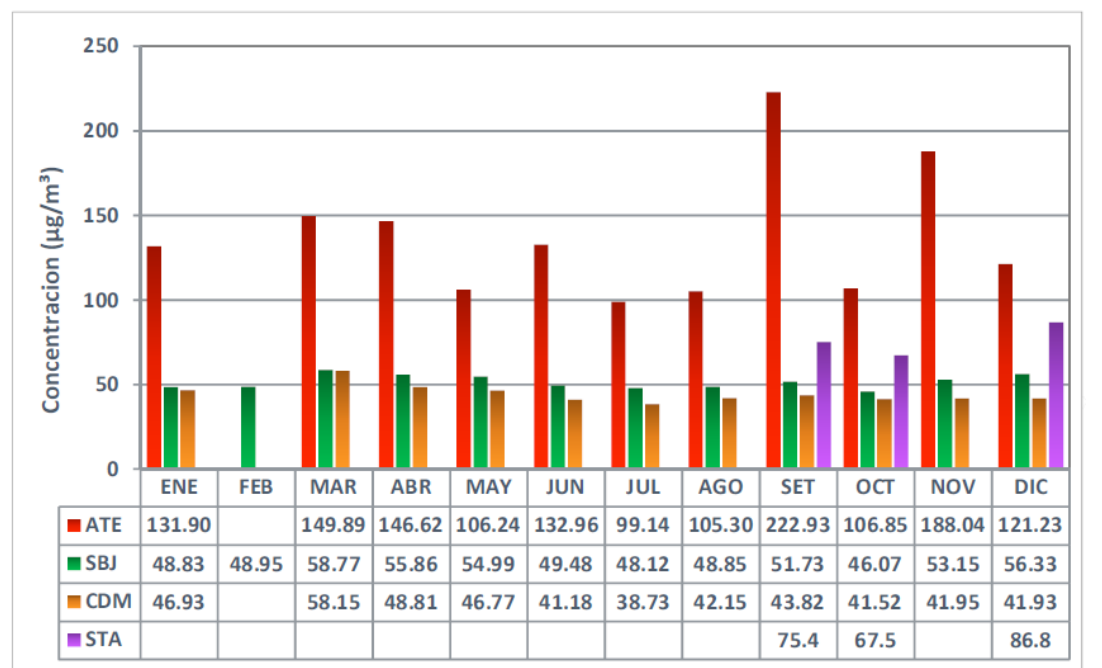

Figura 3. Concentración Media mensual de PM10, en el año 2011, en las estaciones de SENAMHI

También SENAMHI verifica que se cumplan los Estándares Nacionales de Calidad del Aire (ECA) establecidos por el Decreto Supremo 003-2017-MINAM (Ver Tabla 3).

\begin{tabular}{|c|c|c|c|c|}
\hline Parámetros & Período & Valor $\left[\mu \mathrm{g} / \mathrm{m}_{3}\right]$ & Criterios de evaluación & Método de análisis $[1]$ \\
\hline Benceno $\left(\mathrm{C}_{6} \mathrm{H}_{6}\right)$ & Anual & 2 & Media aritmética anual & Cromatografía de gases \\
\hline Dióxido de Azufre $\left(\mathrm{SO}_{2}\right)$ & 24 horas & 250 & NE más de 7 veces al año & $\begin{array}{l}\text { Fluorescencia ultravioleta } \\
\text { (Método automático) }\end{array}$ \\
\hline \multirow{2}{*}{ Dióxido de Nitrógeno $\left(\mathrm{NO}_{2}\right)$} & 1 hora & 200 & NE más de 24 veces al año & \multirow{2}{*}{$\begin{array}{l}\text { Quimioluminiscencia } \\
\text { (Método automático) }\end{array}$} \\
\hline & Anual & 100 & Media aritmética anual & \\
\hline Material Particulado con diámetro & 24 horas & 50 & NE más de 7 veces al año & \multirow{2}{*}{$\begin{array}{l}\text { Separación inercial/filtración } \\
\text { (Gravimetría) }\end{array}$} \\
\hline menor a 2,5 micras $\left(\mathrm{PM}_{2,5}\right)$ & Anual & 25 & Media aritmética anual & \\
\hline Material Particulado con diámetro & 24 horas & 100 & NE más de 7 veces al año & \multirow{2}{*}{$\begin{array}{l}\text { Separación inercial/filtración } \\
\text { (Gravimetría) }\end{array}$} \\
\hline menor a 10 micras $\left(\mathrm{PM}_{10}\right)$ & Anual & 50 & Media aritmética anual & \\
\hline
\end{tabular}

Tabla 3. Estándares de Calidad Ambiental para Aire, ECA-MINAM.

Los motores de combustión interna de los vehículos emiten varios tipos de gases y partículas que contaminan el medio ambiente. Los productos que en mayor cantidad se emiten, son: monóxido de carbono (CO), dióxido de azufre $\left(\mathrm{SO}_{2}\right)$, sulfuro de hidrógeno
$\left(\mathrm{H}_{2} \mathrm{~S}\right)$ y también macropartículas $\left(\mathrm{PM}_{10}\right)$ - SENAMHI basado en el Decreto Supremo $\mathrm{N}^{\circ}$ 009-2003-SA y el Decreto Supremo $\mathrm{N}^{\circ}$ 012-2005-SA, elaboró Tabla de Estados de Alerta Nacionales para contaminantes del aire. Ver Tabla 4.

Tabla 4. Estado de Alerta Nacionales para Contaminantes del Aire. Fuente= SENAMHI

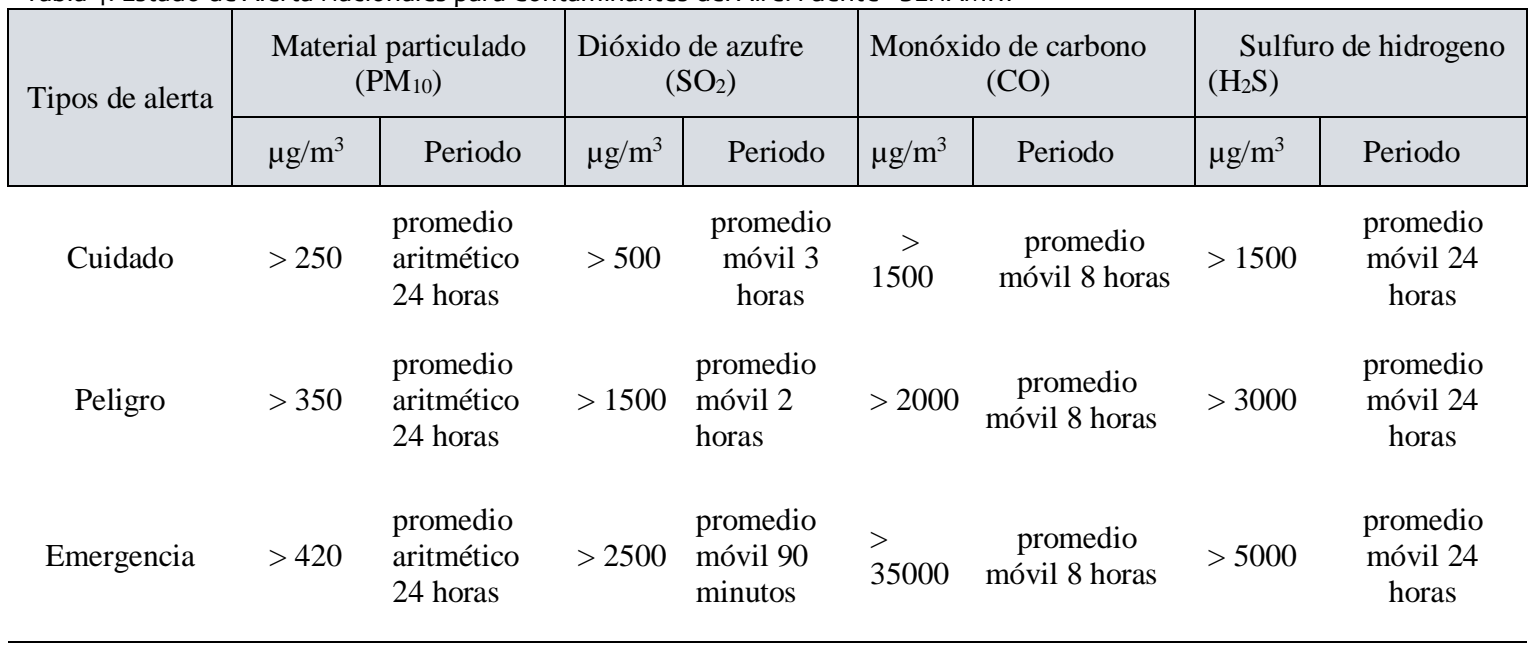



Emisión de Monóxido de Carbono (CO) en Lima
Metropolitana

El CO se genera por combustión incompleta en el motor de un vehículo. La emisión de $\mathrm{CO}$ generada por el parque automotor de Lima (ver Tabla 5) está en un rango de $\left(2845.5\right.$ - 33.8) $\mu \mathrm{g} / \mathrm{m}^{3}$ convertimos a $\mathrm{ppm}=$ (2.489 - 0.0296) ppm. El índice de CO permitido es de $0.1 \mathrm{ppm}$, valores superiores producen síntomas como dolor de cabeza, náusea y fatiga.

Tabla 5. Emisión de CO en Lima Metropolitana. Fuente: SENAMHI

\begin{tabular}{|c|c|c|c|c|c|c|c|c|c|c|}
\hline \multicolumn{11}{|c|}{ ESTACIONE SDE CALIDAD DE AIRE } \\
\hline 2016 mes & $\begin{array}{l}\text { Limz } \\
\text { Este 1 } \\
\text { (Ate) }\end{array}$ & $\begin{array}{l}\text { Limz } \\
\text { Sur } 1 \\
\text { (S zn } \\
\text { Borjz) }\end{array}$ & $\begin{array}{c}\text { Lima Centro } \\
\text { (Jesús Maria- } \\
\text { Campo de } \\
\text { Marte) }\end{array}$ & $\begin{array}{l}\text { Lima } \\
\text { Este } 2 \\
\text { (S zntz } \\
\text { Anita) }\end{array}$ & $\left|\begin{array}{c}\text { Lima Sur } \\
2 \text { (Vitla } \\
\text { Mariz de1 } \\
\text { Trinnfo) }\end{array}\right|$ & $\begin{array}{c}\text { Lima Este } \\
3 \\
\text { (Huachipa) }\end{array}$ & $\begin{array}{c}\text { Lima Este } 4 \\
\text { (San Juan } \\
\text { de } \\
\text { Lurigancho) }\end{array}$ & $\begin{array}{c}\text { Lima } \\
\text { Norte 1 } \\
\text { (San } \\
\text { Martin de } \\
\text { Porres) }\end{array}$ & $\begin{array}{c}\text { Lima Norte } \\
2 \\
\text { (C arzbayllo) }\end{array}$ & $\begin{array}{c}\text { Lima Norte } \\
3 \text { (Pvente } \\
\text { Piedra) }\end{array}$ \\
\hline Enero & $\ldots$ & 642,2 & 264,4 & 856,8 & 600,9 & 181,3 & 294,9 & 1189,5 & 665,4 & 1268,7 \\
\hline Febrero & $\ldots$ & 641,1 & 325,4 & $\ldots$ & 586,1 & 393,5 & 574,7 & 1039,9 & 598,4 & 1378,0 \\
\hline Marzo & 1088,8 & & 358,5 & 952,2 & 670,7 & 655,3 & 420,4 & 1038,2 & 632,3 & 488,1 \\
\hline Abril & 904,2 & & 328,9 & 1069,5 & $\ldots$ & 633,1 & 711,8 & 948,9 & 349,7 & 701,5 \\
\hline Mayo & 2360,2 & $\ldots$ & 333,8 & 1179,8 & 880,2 & 755,6 & 903,2 & 959,6 & 392,8 & 712,7 \\
\hline Innio & 2845.5 & & 3493 & 1056.5 & 903.1 & & 918.5 & & 876.4 & \\
\hline \multicolumn{11}{|c|}{ Variación porcentual } \\
\hline $\begin{array}{l}\text { Respecto } \\
21 \text { mes } \\
\text { anterior }\end{array}$ & 20,6 & $\cdots$ & 4,6 & -10.5 & 2,6 & $\ldots$ & 1.7 & $\ldots$ & 123,1 & $\cdots$ \\
\hline
\end{tabular}

F CA Nacional $10000 \mathrm{ug} / \mathrm{m}^{3}$

Emisión de Dióxido de Azufre $\left(\mathrm{SO}_{2}\right)$ en Lima Metropolitana.

El dióxido de azufre $\left(\mathrm{SO}_{2}\right)$ es un gas pesado, incoloro e inodoro en concentraciones bajas y de color ocre en concentraciones altas. La emisión de $\mathrm{SO}_{2}$ generada por el parque automotor de Lima como se indica en la Tabla 6 está en un rango de $(56,9-1,9) \mu g / \mathrm{m}^{3}$. El índice más bajo registrado en Lima el 2016 fue en abril (estación Campo de Marte - Jesús María), mientras el más alto fue en Mayo (Estación - Ate). El índice de $\mathrm{SO}_{2}$ permitido es de $20 \mu \mathrm{g} / \mathrm{m}^{3}$ (OMS)

Tabla 6. Emisión de $\mathrm{SO}_{2}$ en Lima Metropolitana. Fuente: SENAMHI

\begin{tabular}{|c|c|c|c|c|c|c|c|c|c|c|}
\hline \multicolumn{11}{|c|}{ ESTACIONES DE CALIDAD DE AIRE } \\
\hline 2016 Mes & $\begin{array}{c}\text { Lima Este } 1 \\
\text { (Ate) }\end{array}$ & $\begin{array}{l}\text { Lima Sur } 1 \\
\text { (San Borja) }\end{array}$ & $\begin{array}{l}\text { Lima Centro } \\
\text { (Jesús Maria- } \\
\text { Campo de } \\
\text { Marte) }\end{array}$ & $\begin{array}{c}\text { Lima Este } 2 \\
\text { (Santa Anita) }\end{array}$ & $\begin{array}{l}\text { Lima Sur } 2 \\
\text { (Villa Maria } \\
\text { de1 Triunfo) }\end{array}$ & $\begin{array}{l}\text { Lima Este } 3 \\
\text { (Huachipa) }\end{array}$ & $\begin{array}{l}\text { Lima Este } 4 \\
\text { (San Juan de } \\
\text { L urigancho) }\end{array}$ & $\begin{array}{c}\text { Lima Norte } 1 \\
\text { (San Martin } \\
\text { de Porres) }\end{array}$ & $\begin{array}{l}\text { Lima Norte } 2 \\
\text { (Carabay } 110)\end{array}$ & $\begin{array}{c}\text { Lima Norte } 3 \\
\text { (Pvente } \\
\text { Piedra) }\end{array}$ \\
\hline Enero & $\cdots$ & $\ldots$ & ... & 5,4 & 4,7 & 17,0 & 45,2 & 38,8 & 7,2 & 31,7 \\
\hline Febrero & $\ldots$ & 6,3 & $\ldots$ & $\ldots$ & 6,4 & 22,8 & 8,9 & 36,4 & 6,9 & 11,0 \\
\hline Marzo & 9,2 & 7,4 & ... & $\ldots$ & $\ldots$ & 21,5 & 6,4 & ... & 4,9 & 17,0 \\
\hline Abril & 27,60 & 8,8 & 1,9 & 10,9 & $\ldots$ & 16,8 & 8,3 & 4,6 & 6,2 & 15,1 \\
\hline Mayo & 56,9 & 13,5 & $\ldots$ & 17,0 & 3,8 & 26,8 & 11,4 & 9,0 & 7,2 & 15,9 \\
\hline Jun io & 30,5 & 16,4 & $\ldots$ & 15,3 & 3,4 & $\ldots$ & 12,8 & $\ldots$ & $\ldots$ & $\ldots$ \\
\hline \multicolumn{11}{|c|}{ Variación porcentual } \\
\hline $\begin{array}{l}\text { Respecto al } \\
\text { mes anterior }\end{array}$ & $-46,4$ & 21,5 & $\cdots$ & -10 & $-10,5$ & $\cdots$ & 12,3 & $\cdots$ & $\cdots$ & $\cdots$ \\
\hline
\end{tabular}

E CA Nacional: $10000 \mathrm{ug} / \mathrm{m} 3$

Emisión de Dióxido de Nitrógeno $\left(\mathrm{NO}_{2}\right)$ en Lima Metropolitana.

El dióxido de nitrógeno $\left(\mathrm{NO}_{2}\right)$ es un gas de color marrón claro o amarillo, producido por la quema de combustibles a altas temperaturas, como es el caso de las termoeléctricas, plantas industriales y la combustión del parque automotor. La emisión de $\mathrm{NO}_{2}$ generado por el parque automotor de Lima como se indica en la Tabla 7 está en un rango de $(91.7-4.0) \mu \mathrm{g} / \mathrm{m}^{3}$. El índice más bajo registrado en Lima el 2016 fue en Junio (Estación Santa Anita) mientras el más alto fue en Junio (Estación Ate). El índice de $\mathrm{NO}_{2}$ permitido es de $40 \mu \mathrm{g} / \mathrm{m}^{3}$, OMS. 
Tabla 7. Emisión de $\mathrm{NO}_{2}$ en Lima Metropolitana.

\begin{tabular}{|c|c|c|c|c|c|c|c|c|c|c|}
\hline \multicolumn{11}{|c|}{ ESTACIONES DE CALIDAD DE AIRE } \\
\hline $\begin{array}{l}2016 \\
\text { Mes }\end{array}$ & $\begin{array}{l}\text { Lima } \\
\text { Este } 1 \\
\text { (Ate) }\end{array}$ & $\begin{array}{c}\text { Lima } \\
\text { Sur } 1 \\
\text { (San } \\
\text { Borja) }\end{array}$ & $\begin{array}{l}\text { Lima Centro } \\
\text { (Jesús } \\
\text { María- } \\
\text { Campo de } \\
\text { Marte) }\end{array}$ & $\begin{array}{l}\text { Lima } \\
\text { Este } 2 \\
\text { (Santa } \\
\text { Anita) }\end{array}$ & $\begin{array}{l}\text { Lima Sur } \\
2 \text { (Villa } \\
\text { María } \\
\text { del } \\
\text { Triunfo) }\end{array}$ & $\begin{array}{c}\text { Lima Este } \\
3 \\
\text { (Huachipa) }\end{array}$ & $\begin{array}{c}\text { Lima Este } 4 \\
\text { (San Juan } \\
\text { de } \\
\text { Lurigancho) }\end{array}$ & $\begin{array}{l}\text { Lima } \\
\text { Norte 1 } \\
\text { (San } \\
\text { Martín } \\
\text { de } \\
\text { Porres) }\end{array}$ & $\begin{array}{c}\text { Lima Norte } \\
2 \\
\text { (Carabayllo) }\end{array}$ & $\begin{array}{l}\text { Lima } \\
\text { Norte } 3 \\
\text { (Puente } \\
\text { Piedra) }\end{array}$ \\
\hline Enero & $\ldots$ & $\ldots$ & 58,8 & $\ldots$ & 6,7 & 20,7 & $\ldots$ & 14,8 & 11,4 & 40,3 \\
\hline Febrero & $\ldots$ & 14,0 & 42,9 & $\ldots$ & 13,7 & 23,1 & 20,9 & 13,4 & 11,4 & 36,1 \\
\hline Marzo & 37,4 & 16,1 & 11,9 & 4,6 & 22,9 & 24,9 & 24,7 & 15,8 & 20,5 & 37,1 \\
\hline Abril & 51,8 & 20,6 & 23,9 & 7,6 & $\ldots$ & 29,2 & 38,9 & 19,2 & 24,5 & 32,3 \\
\hline Mayo & 65,7 & 39,2 & 30,3 & 5,3 & 24,7 & 34,1 & 54,2 & 24,7 & 32,7 & 34,7 \\
\hline Junio & 91,7 & 48 & 35,6 & 4,0 & 24,8 & $\ldots$ & 63,1 & $\ldots$ & 34,1 & $\ldots$ \\
\hline \multicolumn{11}{|c|}{ Variación porcentual } \\
\hline $\begin{array}{l}\text { Respecto } \\
\text { al mes } \\
\text { anterior }\end{array}$ & 39,6 & 22,4 & 17,5 & $-24,5$ & 0,4 & $\ldots$ & 16,4 & $\ldots$ & 4,3 & ... \\
\hline
\end{tabular}

ECA Nacional: $10000 \mathrm{ug} / \mathrm{m} 3$

ug/m3: Microgramo por metro cúbico.

Fuente: Servicio Nacional de Meteorología e Hidrología (SENAMHI)-Dirección General de Investigación y Asuntos Ambientales.

La Organización Mundial de la Salud (OMS) elaboró en el 2016 un nuevo modelo de calidad del aire que confirma que el $92 \%$ de la Población Mundial vive en lugares donde los niveles de calidad del aire exceden los límites fijados por este organismo. Las guías de calidad del aire de la OMS tienen por objeto ofrecer orientación sobre la manera de reducir los efectos de la contaminación del aire en la salud. Ver Tabla 8.

Tabla 8. Normas de Calidad del Aire de la OMS en diferentes países

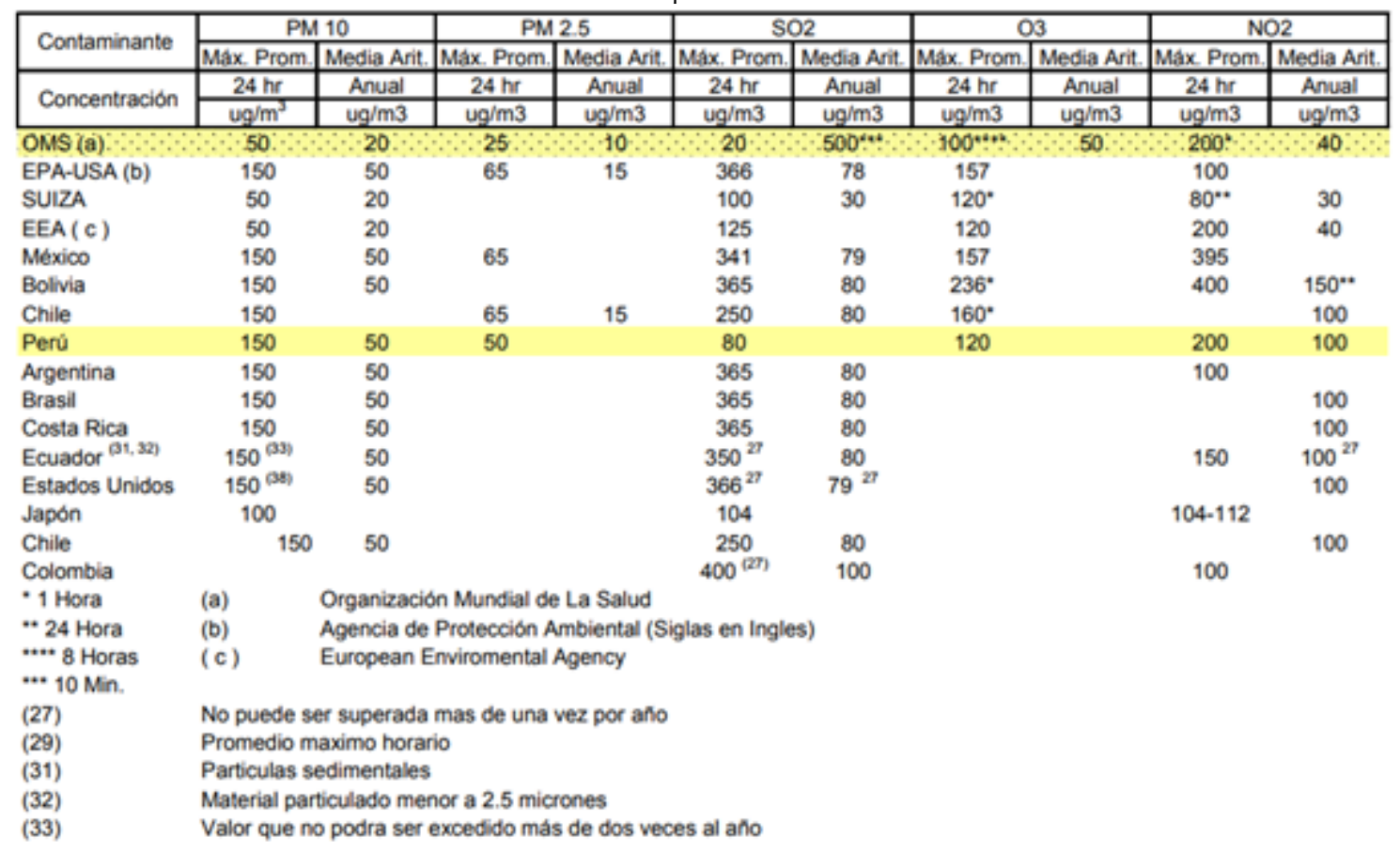

Fuente: Ministerio de Salud (MINSA)-Dirección General de Salud Ambiental (DIGESA).(2011) 


\section{CONCLUSIONES}

Mediante el proyecto formativo elaborado, se demostró que los contenidos del sílabo del curso de Física General II (leyes de la termodinámica) sirven de base para elaborar el sílabo del curso basado en competencias.

Los resultados demostraron que el parque automotor de Lima se estima en 1752919 vehículos en el año 2016 (MTC); su evolución de 957368 a 1752919 vehículos entre los años 2007-2016 (SUNARP). Además la edad promedio del parque automotor de Lima es de 22,5 años para el servicio público y del privado 15,5 años. Esto ha generado el agravamiento de la contaminación ambiental.

La emisión de gases contaminantes generados por el parque automotor de Lima (SENAMHI) es:

CO: $(2,489-0,0296)$ ppm. El índice permitido es de 0,1 ppm (OMS).

$\mathrm{SO}_{2}:(56,6-1,9) \mu \mathrm{g} / \mathrm{m}^{3}$ el índice es permitido es de 20 $\mu \mathrm{g} / \mathrm{m}^{3}$ (OMS).

$\mathrm{NO}_{2}:(91,7-4,0) \mu \mathrm{g} / \mathrm{m}^{3}$ el índice es permitido es de 40 $\mu \mathrm{g} / \mathrm{m}^{3}$ (OMS).

Los índices de contaminación encontrados de Monóxido de Carbono (CO), dióxido de azufre $\left(\mathrm{SO}_{2}\right)$, dióxido de Nitrógeno y las altas concentraciones de material particulado menor a 10 micrómetros $\left(\mathrm{PM}_{10}\right)$, nos permiten a nivel de la población de lima, plantear una cultura en termodinámica para mejorar la situación del parque automotor y bajar los índices de contaminación del aire.

Una cultura que involucre los siguientes aspectos:

1. Reordenamiento del tránsito vehicular.

2. Construcción del medio de transporte masivo.

3. Impulsar una cultura en termodinámica en la población.

4. Renovación del parque automotor.

\section{REFERENCES}

[1] Tallman D E, Wallace G G 1997 Synth. Met. 9013

[2] Kroto H W, Fischer J E, Cox D E 1993 The Fullerenes Pergamon:Oxford

[3] MacDiarmid A G, Epstein A J 1991 in ed.W R Salaneck, D T Clark, E J Samuelson Science and Applications of Conducting Polymers Adam Hilger: Bristol p.117

[4] Eaton D I 1975 Porous glass support material US Patent No. 3904 